\title{
Molecular dynamics simulations of the freezing of gold nanoparticles
}

\author{
Y. Chushak ${ }^{\mathrm{a}}$ and L.S. Bartell \\ Department of Chemistry, University of Michigan, Ann Arbor, MI48109, USA
}

Received 6 November 2000

\begin{abstract}
A set of molten gold clusters, each with 1157 gold atoms, was studied by molecular dynamics simulations as the clusters underwent freezing at three different temperatures. Most of the clusters attained an icosahedral structure upon freezing, a structure found to be stable to mild annealing. Other structures observed were imperfect truncated decahedral, truncated octahedral and hexagonal close packed structures. The role of kinetics in the process of cluster solidification is discussed.
\end{abstract}

PACS. 36.40.Ei Phase transitions in clusters - 61.46. $+\mathrm{w}$ Nanoscale materials: clusters, nanoparticles, nanotubes, and nanocrystals

\section{Introduction}

Over the past three decades a considerable experimental and theoretical effort has been devoted to the study of the structure of metal clusters and its size dependence. Various experimental techniques have been applied to determine the atomic arrangements in clusters [1]. In diffraction experiments, which probe an ensemble of clusters, the weak scattering from individual particles is compensated by the ensemble size. However, it is practically impossible to prepare a large ensemble of nanoparticles with a narrow size distribution. Furthermore, even for a given particle size, a distribution of structural isomers is observed. Therefore, the interpretation of diffraction patterns of such mixtures can be very complicated $[2,3]$.

High resolution electron microscopy (HREM) has the advantage that individual nanoparticles deposited on a supporting substrate can be directly observed. HREM can resolve atomic planes in nanoparticles and has been used extensively to investigate their structure [4-6]. HREM observations on gold particles reveal a rich diversity in the structures obtained. The main types identified have been face-centered cubic (FCC) cuboctahedra (CO), twined FCC (containing one or several parallel twin planes) [5], twined hexagonal close packed (HCP) [8], icosahedral and truncated icosahedral [4,7] (Ico), and truncated decahedral (Dh). However, HREM yields projected images, and the correct interpretation of these images requires computer simulations. Many particles observed have yet to be identified. In most cases, the particles were prepared on a substrate by evaporation methods in which kinetics plays a major role, equilibrium structures not always having been achieved. This has led to controversies about what

\footnotetext{
a e-mail: chushak@umich.edu
}

the true equilibrium forms are. Furthermore, the high energy electron flux needed to obtain images in HREM, can heat the particles and may cause structural rearrangements, especially for small particles [9].

Computational experiments are uniquely suited for studying the internal organization of nanoparticles. Modern computers can readily simulate nanometer-size particles on a nanosecond time scale if a realistic interatomic potential function is available. The majority of calculations have been focused on an exhaustive search of the lowest energy configuration (global minimum on the energy surface) and its size dependence. Recently, Cleveland et al. $[10,11]$ have used an embedded atom (EAM) potential to determine the optimal structure of nanometersize $\mathrm{Au}$ clusters comprised of 100 to 1000 atoms (1.4-3.0 nm in diameter). A close competition among several structural types was found across the entire range of sizes. The predominant types were truncated decahedral, truncated octahedral (TO+) and its symmetrically twinfaulted variant $(\mathrm{t}-\mathrm{TO}+)$. Similar result was obtained by Baletto et al. [12] using quenched molecular dynamics simulations with a potential function developed on the basis of the second-moment approximation to the tight-binding model. All other configurations, including the icosahedral which is the lowest-energy configuration for $\mathrm{Ni}$ or $\mathrm{Cu}$ clusters in the same size range [13], are much less stable. On the other hand, it was found [14] that, during heating, gold nanoparticles initially possessing their stable, lowtemperature structures underwent a solid state transformation to an icosahedral structure before melting.

In the present paper we report the results of molecular dynamics simulations of the solidification of molten gold clusters. To better understand the process of crystallization while, at the same time, acquiring sets to enable a determination of nucleation rates, we performed 
Table 1. Bond-order parameters for different local structures.

\begin{tabular}{lccc}
\hline structure & $q_{4}$ & $q_{6}$ & $q_{8}$ \\
\hline FCC & 0.191 & 0.575 & 0.404 \\
HCP & 0.097 & 0.485 & 0.317 \\
Ico & 0.0 & 0.663 & 0.0 \\
Dh & 0.053 & 0.430 & 0.139 \\
\hline
\end{tabular}

simulations at each of three different temperatures on a set of 20 molten clusters of the same size, 1157 atoms. Even though the individual clusters were generated and frozen under identical conditions, several different final structures were obtained. These will be discussed in the following.

\section{Details of computer simulations}

Molecular dynamics simulations were performed on gold clusters containing 1157 atoms represented by an EAM interaction potential [15]. Equations of motion were integrated by a fifth-order Gear predictor-corrector algorithm with a time step of $2.8 \mathrm{fs}$ [16]. Simulations were carried out at constant temperature with a tolerance $\Delta T=5 \mathrm{~K}$. An initial, approximately spherical FCC cluster was heated up from $300 \mathrm{~K}$ to $1200 \mathrm{~K}$ (well above the cluster's melting temperature $T_{m}=920 \mathrm{~K}$ ) to ensure its complete melting. The configuration at $1200 \mathrm{~K}$ was additionally equilibrated and 20 configurations were saved, one every $7 \mathrm{ps}$. These clusters, cooled to $750 \mathrm{~K}$ at a cooling rate of $15 \times 10^{12} \mathrm{~K} / \mathrm{s}$, served to generate starting configurations for crystallization. Production runs were carried out at three temperatures: $740 \mathrm{~K}, 720 \mathrm{~K}$ and $700 \mathrm{~K}$. Durations of runs were $1 \mathrm{~ns}$. The frozen clusters materializing were cooled to $300 \mathrm{~K}$ at a cooling rate of $3 \times 10^{11} \mathrm{~K} / \mathrm{s}$ for further annealing. Phase transitions were recognized by the evolution of configuration energy and by the structure analyses described in the next section.

\section{Structure analysis}

In the present study we used the bond-order parameter method $[17,18]$ to distinguish between the liquid and solid atoms as well as to identify the clusters' structures. We define "bonds" in terms of unit vectors $\boldsymbol{r}_{i j}$ connecting an atom $i$ with its neighboring atoms $j$ that are within a given radius $r_{c u t}$ of $i$. The orientation of a bond $\boldsymbol{r}_{i j}$ with respect to some reference system is specified by the spherical harmonics $Y_{l m}\left(\boldsymbol{r}_{i j}\right)=Y_{l m}\left(\theta_{i j} ; \varphi_{i j}\right)$, where $\theta_{i j}$ and $\varphi_{i j}$ are the polar and azimuthal angles. Only even- $l$ spherical harmonics, which are invariant under inversion, were considered. The local order around an atom $i$ is determined
Table 2. Distribution of final configurations $v s$. freezing temperature.

\begin{tabular}{lccc}
\hline & \multicolumn{3}{c}{ freezing temperature } \\
\cline { 2 - 4 } structure & $700 \mathrm{~K}$ & $720 \mathrm{~K}$ & $740 \mathrm{~K}$ \\
\hline Ico & 12 & 13 & 12 \\
Dh & 2 & 1 & 4 \\
TO & 6 & 4 & 3 \\
HCP & - & 2 & - \\
\hline
\end{tabular}

by averaging over all bonds with its neighbors $N_{n b}(i)$

$$
q_{l m}(i)=\frac{1}{N_{n b}(i)} \sum_{j=1}^{N_{n b}(i)} Y_{l m}\left(\boldsymbol{r}_{i j}\right) .
$$

To avoid a dependence of the local order parameter on the choice of reference system, a second-order invariant is constructed [17]

$$
q_{l}(i)=\left[\frac{4 \pi}{2 l+1} \sum_{m=-l}^{l}\left|q_{l m}(i)\right|^{2}\right]^{1 / 2}
$$

The first nonzero value of $q_{l}(i)$ (other than constant for $l=0$ ) occurs at $l=4$ for atoms with cubic or decahedral local symmetry and at $l=6$ in aggregates with icosahedral symmetry. The values of bond-order parameters for different structures, listed in Table 1, were used to identify the structure of each cluster produced.

The structure of a cluster is also reflected in its diffraction pattern. We have used the Debye scattering equation [19] to calculate the X-ray diffraction intensity $I(s)$ corresponding to a rotationally averaged ensemble as a function of the scattering variable $s=2 \sin (\Theta) / \lambda$

$$
I(s)=f^{2}(s) \sum_{i, j} \frac{\sin \left(2 \pi s r_{i j}\right)}{2 \pi s r_{i j}}
$$

where $r_{i j}$ is the distance between atoms $i$ and $j$ in the cluster, $f(s)$ is the atomic scattering factor, and $\Theta$, the Bragg angle.

\section{Results}

Results of the runs to freeze clusters are summarized in Table 2. Only in one run, one at $740 \mathrm{~K}$, did a cluster fail to freeze. Clearly the icosahedral structure formed preferentially but others also formed spontaneously. Figure 1 shows typical structures obtained during the freezing of supercooled clusters. The dark grey spheres represent gold atoms with an FCC local structure and light grey spheres are atoms with an HCP environment. In the case of Ico or Dh structures HCP atoms are located in the twin boundary of FCC tetrahedra with (111) faces. Stacking faults in 


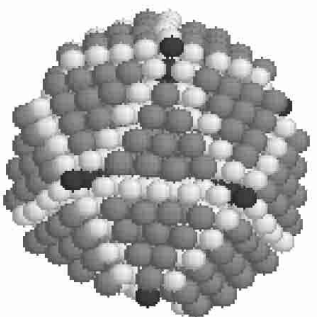

Ico

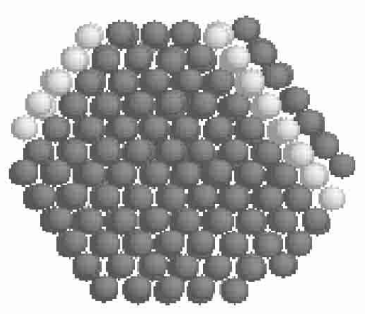

TO

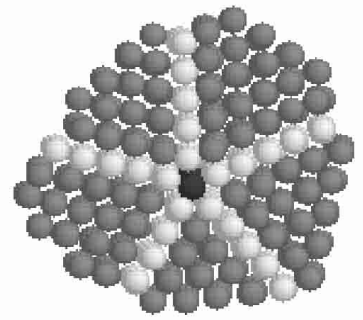

$\mathrm{Dh}$

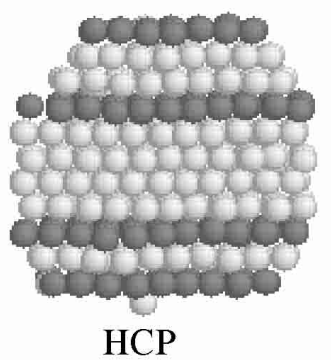

Fig. 1. Typical structures materializing spontaneously during the solidification of supercooled gold clusters. Dark grey spheres represent gold atoms with an FCC local structure, light grey spheres are atoms in an HCP environment, and black atoms indicate sites with 5-fold (Dh) symmetry.

the FCC planes of TO clusters cause the planes identified as HCP. Correspondingly, the stacking faults of planes in the HCP structure lead to the appearance of planes with FCC local symmetry.

The X-ray diffraction (XRD) powder patterns for clusters with various structures are presented in Figure 2. The XRD pattern for clusters with HCP symmetry has a welldefined three-peak feature that affords it ready identification. The diffraction pattern of TO clusters also has well-defined peaks, which can be indexed as Bragg reflections from planes in an FCC lattice. XRD reflections from Ico and Dh clusters are weaker and much more diffuse owing to the fact that domains with ordered structures are smaller because of the multiple twinning.

Finally, Figure 3 shows how the configurational energy varies with temperature for clusters with different structures. Clearly, all solid clusters obtained from freezing have very similar configurational energies, despite conspicuous differences in their shapes. What is striking is that their energies are much lower than the energy of the initial quasispherical FCC cluster, the favored structure of bulk gold. Only after heating to $600 \mathrm{~K}$ has the FCC structure rearranged sufficiently for its configurational energy to approach that of the forms generated on freezing.

\section{Discussion}

Intensive computer investigations [10-12,20] which sought the optimal structures for gold clusters have indicated that the icosahedral structure is energetically noncompetitive even in the very small size range [20]. As the size of a

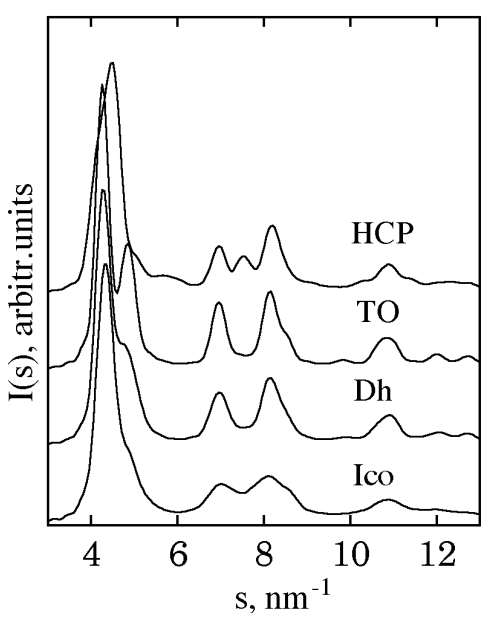

Fig. 2. Calculated X-ray diffraction powder patterns of clusters with different structures at $T=300 \mathrm{~K}$.

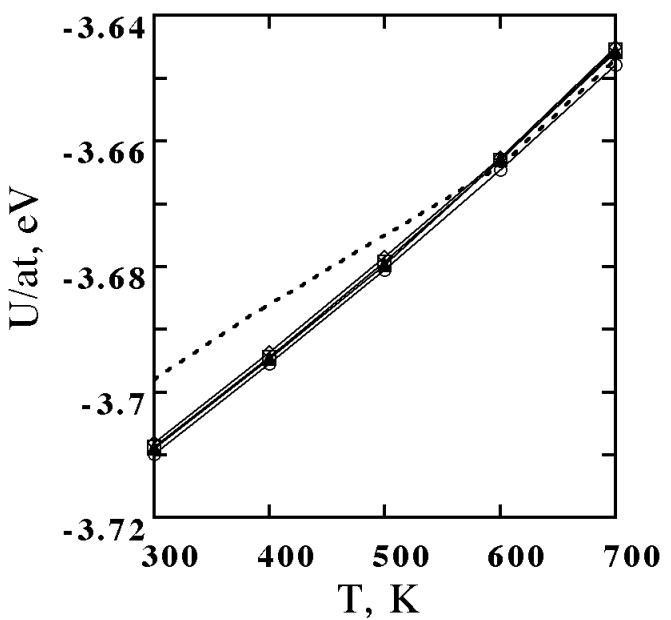

Fig. 3. Configurational energies of clusters with different structures as a function of temperature during cooling (solid lines) and during the heating of a cluster initially possessing an FCC structure (dashed curve). Markers, which severely overlap because of the close similarity of energies of the different atomic packings of the systems plotted, correspond to structures dominated by ico - solid triangles, Dh - diamonds, TO open circles, and HCP - open squares.

cluster increases this structure was calculated to become less and less favorable due to accumulated strain energy, especially at the center of a cluster. On the other hand, icosahedral gold nanoparticles generated in the laboratory by various methods, have been observed in HREM studies in the 1960's by Komoda [4] and Allpress et al. [21], in the 1980's by Marks and Smith [22] and in the 1990's by Buffat et al. [5] and Jose-Yacamán et al. [7]. In our own computer experiments, most of the 60 clusters studied froze to an icosahedral structure. One could argue that it is not an equilibrium structure due to the limited time of computer simulations. We performed further annealing of clusters by relatively slow cooling to $300 \mathrm{~K}$. This treatment did 
not induce icosahedral clusters to transform to any of the other possible low-energy structures. Very recently a new method for obtaining free gold nanoparticles - bioprecipitation - was discovered [23]. The growth mechanism is so slow in this process that the structures produced are presumably at equilibrium. An analysis of the frequency of appearance of different structures showed that icosahedral is the most frequently formed structure for gold particles up to $70 \mathrm{~nm}$ in diameter.

Another striking result of our simulations was the formation of nanoparticles with a predominantly HCP structure. Such a structure was also observed in experimental investigations $[8,23]$. This is of interest because the HCP structure had not been considered to be stable. We have observed a transition from HCP to FCC structure in clusters of a larger size. Nevertheless, two clusters with 1157 atoms remained in a principally HCP arrangement even during cooling, indicating the presence of a significant energy barrier for the HCP-FCC transformation.

Clearly, the kinetics of nucleation and growth in clusters plays a key role in determining the structure of clusters. Different structures were obtained under identical conditions due to the stochastic nature of nucleation.

This research was supported by a grant from the National Science Foundation.

\section{References}

1. L.D. Marks, Rep. Progr. Phys. 57, 603 (1994).

2. D. Reinhard, B.D. Hall, P. Berthoud, S. Valkealahti, R. Monot, Phys. Rev. B 58, 4917 (1998).

3. B.D. Hall, J. Appl. Phys. 87, 1666 (2000).

4. T. Komoda, Jpn J. Appl. Phys. 7, 27 (1968).

5. P.-A. Buffat, M. Flueli, R. Spycher, P. Stadelmann, J.P. Borel, Faraday Discuss. 92, 173 (1991).
6. J.A. Ascencio, C. Gutiérrez-Wing, M.E. Espinosa, M. Marin, S. Tehuacanero, C. Zorrilla, M. José-Yacamán, Surf. Sci. 396, 349 (1998).

7. J.A. Ascencio, M. Pérez, M. José-Yacamán, Surf. Sci. 447, 73 (2000).

8. M. José-Yacamán, R. Herrera, A. Gómez, S. Tehuacanero, P. Schabes-Retchkiman, Surf. Sci. 237, 248 (1990).

9. D.J. Smith, A.K. Petford-Long, L.R. Wallenberg, J.-O. Bovin, Science 233, 872 (1986).

10. C.L. Cleveland, U. Landman, T.S. Schaaff, M.N. Shafigullin, P.W. Stephens, R.L. Whetten, Phys. Rev. Lett. 79, 1873 (1997).

11. C.L. Cleveland, U. Landman, M.N. Shafigullin, P.W. Stephens, R.L. Whetten, Z. Phys. D 40, 503 (1997).

12. F. Baletto, C. Mottet, R. Ferrando, Surf. Sci. 446, 31 (2000).

13. C.L. Cleveland, U. Landman, J. Chem. Phys. 94, 7376 (1991).

14. C.L. Cleveland, W.D. Luedtke, U. Landman, Phys. Rev. B 60, 5965 (1999).

15. S.M. Foiles, M.I. Baskes, M.S. Daw, Phys. Rev. B 33, 7983 (1986).

16. The Classical Molecular Dynamics code (AL_CMD) developed at Ames Laboratory of the U.S. Department of Energy has been used in present simulations.

17. P.J. Steinhard, D.R. Nelson, M. Ronchetti, Phys. Rev. B 28, 784 (1983).

18. Y. Chushak, L.S. Bartell, J. Phys. Chem. A 104, 9328 (2000).

19. B.E. Warren, X-Ray Diffraction (Dover, New York, 1990).

20. K. Michaelian, N. Rendon, I.L. Garzon, Phys. Rev. B 60, 2000 (1999).

21. T.G. Allpress, T.V. Sanders, Philos. Mag. 10, 654 (1964).

22. L.D. Marks, D. Smith, J. Cryst. Growth 54, 425 (1981).

23. J.L. Gardea-Torresdey, K.J. Tiemann, G. Gamez, K. Dokken, S. Tehuacanero, M. José-Yacamán, J. Nanoparticle Res. 1, 397 (1999). 